\title{
Vitamin D and coronavirus disease 2019 (COVID-19): rapid evidence review
}

\author{
Zahra Raisi-Estabragh ${ }^{1,2} \cdot$ Adrian R. Martineau $^{3}$ - Elizabeth M. Curtis ${ }^{4} \cdot$ Rebecca J. Moon $^{4} \cdot$ Andrea Darling $^{5}$. \\ Susan Lanham-New ${ }^{5}$ Kate A. Ward ${ }^{4,6} \cdot$ Cyrus Cooper $^{4,6,7} \cdot$ Patricia B. Munroe $^{1} \cdot$ Steffen E. Petersen ${ }^{1,2}$. \\ Nicholas C. Harvey ${ }^{4,6}$
}

Received: 2 April 2021 / Accepted: 23 May 2021 / Published online: 12 June 2021

(c) The Author(s) 2021

\begin{abstract}
Background The rapid global spread of severe acute respiratory syndrome coronavirus 2 (SARS-CoV-2), the virus that causes coronavirus disease 2019 (COVID-19), has re-ignited interest in the possible role of vitamin D in modulation of host responses to respiratory pathogens. Indeed, vitamin D supplementation has been proposed as a potential preventative or therapeutic strategy. Recommendations for any intervention, particularly in the context of a potentially fatal pandemic infection, should be strictly based on clinically informed appraisal of the evidence base. In this narrative review, we examine current evidence relating to vitamin D and COVID-19 and consider the most appropriate practical recommendations.

Observations Although there are a growing number of studies investigating the links between vitamin D and COVID-19, they are mostly small and observational with high risk of bias, residual confounding, and reverse causality. Extrapolation of molecular actions of $1,25(\mathrm{OH})_{2}$-vitamin $\mathrm{D}$ to an effect of increased $25(\mathrm{OH})$-vitamin $\mathrm{D}$ as a result of vitamin $\mathrm{D}$ supplementation is generally unfounded, as is the automatic conclusion of causal mechanisms from observational studies linking low $25(\mathrm{OH})$-vitamin $\mathrm{D}$ to incident disease. Efficacy is ideally demonstrated in the context of adequately powered randomised intervention studies, although such approaches may not always be feasible.

Conclusions At present, evidence to support vitamin D supplementation for the prevention or treatment of COVID-19 is inconclusive. In the absence of any further compelling data, adherence to existing national guidance on vitamin D supplementation to prevent vitamin D deficiency, predicated principally on maintaining musculoskeletal health, appears appropriate.
\end{abstract}

Keywords Severe acute respiratory syndrome coronavirus-2 (SARS-CoV-2) · Coronavirus disease 2019 (COVID-19) . Respiratory infection · Vitamin D · Vitamin D deficiency · Osteoporosis · Musculoskeletal health

Nicholas C. Harvey

nch@mrc.soton.ac.uk

1 William Harvey Research Institute, NIHR Barts Biomedical Research Centre, Queen Mary University of London, London, UK

2 Barts Heart Centre, St Bartholomew's Hospital, Barts Health NHS Trust, London, UK

3 Institute of Population Health Sciences, Barts and The London School of Medicine and Dentistry, Queen Mary University of London, London, UK

4 MRC Lifecourse Epidemiology Unit, University of Southampton, Southampton General Hospital, Southampton SO16 6YD, UK
5 Nutrition, Food and Exercise Sciences Department, School of Biosciences and Medicine, Faculty of Health and Medical Sciences, University of Surrey, Guildford, UK

6 NIHR Southampton Biomedical Research Centre, University of Southampton and University Hospital Southampton NHS Foundation Trust, Southampton, UK

7 NIHR Oxford Biomedical Research Centre, University of Oxford, Oxford, UK 


\section{Introduction}

The importance of vitamin D for regulation of calcium and phosphate balance in musculoskeletal physiology is well established [1]. Vitamin D receptors are also expressed by many non-skeletal tissues suggesting a broader role for vitamin $\mathrm{D}$ in human health, particularly in modulating immune system activities [2-7]. Many immune cells have been found to express vitamin $\mathrm{D}$ receptors, including $\mathrm{T}$ and B cells, monocytes, macrophages, and dendritic cells [8]. It has been proposed that vitamin D may augment the first line of defence against invading pathogens and suppress the adaptive immune response, mitigating autoimmune conditions [2]. Indeed, a growing body of evidence suggests multiple biological roles for vitamin D. However, while many observational studies have demonstrated associations between low $25(\mathrm{OH})$-vitamin $\mathrm{D}[25(\mathrm{OH}) \mathrm{D}]$ levels and a wide range of non-musculoskeletal morbidities and increased mortality, results from randomised controlled trials (RCTs) of vitamin D supplementation in these contexts have been less consistent $[9,10]$.

The rapid global spread of coronavirus disease 2019 (COVID-19), which is caused by severe acute respiratory syndrome coronavirus 2 (SARS-CoV-2) infection, has renewed interest in the possible role of vitamin $\mathrm{D}$ in modulating the immune response to respiratory infections. Indeed, widespread vitamin D supplementation has been proposed as a preventative health measure [11]. However, at present the evidence-base is of insufficient quality to support such recommendations.

Importantly, while the active $1,25(\mathrm{OH})_{2}$-vitamin $\mathrm{D}$ form has been shown to have a role within the immune system [12], evidence linking low 25(OH)D levels (the main circulating form, which correlates poorly to $1,25(\mathrm{OH})_{2^{-}}$ vitamin D) with increased risk or severity of COVID-19 has been inconsistent [13]. The non-COVID-19 literature contains numerous examples of $25(\mathrm{OH}) \mathrm{D}$-morbidity associations which could be attributable to confounding or reverse causation [14]. An example, demonstrated using Mendelian randomisation, is of the observational association between low 25(OH)D levels and obesity, which in causal analyses implicate obesity as driving lower $25(\mathrm{OH})$ D rather than the other way round [15]. Furthermore, 25(OH)D levels are determined by sunlight exposure to the skin, supplement use, diet, and comorbidity (since, in some clinical contexts, $25(\mathrm{OH}) \mathrm{D}$ may be a negative acute phase reactant [16-19]). As such, vitamin D status is closely linked to general health and the potential for confounding in observational studies is high [14].

While vitamin $\mathrm{D}$ (as cholecalciferol [vitamin $\mathrm{D}_{3}$ ] or ergocalciferol [vitamin $\mathrm{D}_{2}$ ]) has a wide therapeutic window with a relatively low risk of toxicity, the easy availability of high-dose supplements (particularly on the internet) may increase the likelihood of such rare events in the population [20]. A further consideration in the context of the current pandemic is the potential for adverse health behaviour resulting from a perception of protection gained from taking supplements. Thus, it is important that a strictly evidence-based approach, together with health economic assessment (which is beyond the scope of this review), underpin any health recommendations in relation to the use of vitamin D supplements to prevent or treat COVID-19. In this paper, we review the pertinent literature relating to vitamin $\mathrm{D}$ and respiratory infections, with a particular focus on COVID-19.

\section{Search strategy}

We searched Ovid Medline electronic database (1946 to May 14, 2021) using the following Medical Subject Heading $(\mathrm{MeSH})$ terms combined using Boolean operators: [("COVID-19" OR "SARS-CoV-2" OR "Severe Acute Respiratory Syndrome") AND ("Vitamin D" OR "Vitamin D deficiency")]. To ensure wide breadth of the search, we exploded the MeSH terms and included all subheadings. Papers were selected for inclusion in the review based on title and abstract screening followed by full text review, if appropriate. Further relevant studies were identified through cross referencing and author searches. Ongoing and planned studies were identified from searching https://clinicaltrials. gov. With regards summarising evidence relating to vitamin D and non-COVID-19 acute respiratory infections, we selected the latest and most comprehensive systematic evidence reviews on the topic.

\section{Vitamin $D$ and acute respiratory infections}

The role of vitamin D in acute respiratory infections has been examined in multiple studies, including a number of clinical trials. A recent meta-analysis of stratified aggregate data from 48,488 participants in 43 RCTs testing the effect of vitamin D on the risk of incident acute respiratory infections in adults and children demonstrated a modest protective effect from vitamin D supplementation (OR 0.92, 95\% CI 0.86 to 0.99 ) [21]. There was significant variation in baseline $25(\mathrm{OH}) \mathrm{D}$ status of participants and in supplementation regimens. In many of the included studies, the diagnosis of acute respiratory infection was often ascertained from self-report. Overall, there was high between-study heterogeneity and the possibility of publication bias towards studies reporting a protective effect of supplementation. A second meta-analysis found that vitamin D supplementation did not reduce the risk of acute respiratory infections in $15 \mathrm{RCTs}$ including healthy adults and children [22]. Again, there was 
substantial heterogeneity in the results, which might partly reflect differential effects of the intervention in different populations or differences in study designs. Overall, while the totality of the evidence base suggests the potential for a protective effect of vitamin $\mathrm{D}$ on risk of acute respiratory infections, there is inconsistency across studies and in particular definitive evidence in the older populations, who are most at risk from COVID-19, is still lacking.

\section{Vitamin D and COVID-19}

\section{In vitro studies}

$25(\mathrm{OH}) \mathrm{D}$ is the pre-hormone to the biologically active $1,25(\mathrm{OH})_{2}$-vitamin $\mathrm{D}$, which has recognized in vitro immunomodulatory activity [8]. Mok et al. [23] sought to investigate the role of calcitriol in the context of COVID-19 using animal (monkey) and human (hepatoma, nasal epithelial) cell lines. The authors demonstrate potent activity of calcitriol against SARS-CoV-2 at a cellular level, but whether these finding can be translated into an effect of vitamin D supplementation at daily/bolus doses remains to be studied [23]. Overall, the in vitro data suggest that the vitamin D pathway may be a biologically plausible target in prevention or treatment of COVID-19. This hypothesis has prompted many researchers to examine the relationship between 25(OH)D and COVID-19 outcomes in ecological, observational, and interventional studies.

\section{Ecological studies}

In several early reports, researchers noted that countries with lower national average 25(OH)D levels had higher COVID19 cases per head of population. For instance, Ilie et al. [24] reported a significant negative correlation of average population serum 25(OH)D levels with the number of COVID-19 cases and deaths per million population across 20 European countries. A similar study focused specifically on older individuals across 12 European countries, also reported an un-adjusted association between lower average population $25(\mathrm{OH}) \mathrm{D}$ and greater COVID-19 cases per million population [25]. In another study, Walrand et al. [26] estimated the date of sudden surge of COVID-19 cases (surge date) for 18 European countries using a model fitted based on daily new cases reported over the previous 2 months in Autumn 2020. They reported correlation of country surge date with latitude; specifically, they linked the surge date to when a country's sun-derived UV daily dose dropped to below $34 \%$ of that of $0^{\circ}$ latitude. Furthermore, the authors demonstrated that this might be explained by seasonal drop in $25(\mathrm{OH}) \mathrm{D}$, as estimated using published data on population seasonal variations in vitamin D. In a later study, Singh et al. [27], hypothesised that, given a true protective effect of vitamin
D, correlation between vitamin D levels and COVID-19 outcomes should be strengthened after the infection peak. To investigate, they estimate the association of average national vitamin $\mathrm{D}$ levels (obtained from previously published reports) and publicly available data on COVID-19 cases and deaths in 20 European countries after the first infection peak of COVID-19 [27]. While the authors document a significant negative correlation between vitamin D levels and COVID-19 cases per million population, the association between vitamin D and COVID-19 death was not statistically significant. It is well recognised that such ecological analyses are highly prone to confounding and may not delineate causal associations [28]. Other factors such as population demographics, density of housing, obesity, lifestyle and dietary factors, together with the potential for underlying genetic differences and inconsistency between different assays [29], may all influence such associations.

\section{Observational studies}

Several studies in patient cohorts have compared serum 25(OH)D levels between individuals with and without COVID-19. D'Avolio et al. [30] compared serum 25(OH)D (measured within 7 weeks prior to testing) in patients with a positive COVID-19 test ( $n=27$ ) with those with symptoms of respiratory infection and a negative test $(n=80)$ and with a historic pre-pandemic cohort $(n=1377)$. They report significantly lower $25(\mathrm{OH}) \mathrm{D}$ levels in the test positive cohort compared to the test negative and the historic cohorts. There was no significant difference between $25(\mathrm{OH}) \mathrm{D}$ levels of the test negative and historic groups. In a study of individuals with Parkinson's disease, Fasano et al. [31] report that individuals without COVID-19 were more likely to take vitamin D supplementation compared to those with probable or confirmed COVID-19. These unadjusted associations suggest a potential link between low vitamin D status and increased susceptibility to COVID-19; however, observed associations may be influenced by confounding factors.

Kaufman et al. [32] present a large retrospective observational study using data from a national clinical laboratory database in the Unites States (US). SARS-CoV-2 test results for 191,779 patients were matched to serum $25(\mathrm{OH})$ D results recorded in the preceding 12 months. Geographic latitude and ethnicity were estimated by matching residential ZIP codes to US Census data. Higher serum 25(OH)D was associated with lower rate of SARS-CoV-2 positivity (OR 0.98, 95\% CI 0.98-0.99) in logistic regression models adjusted for age, sex, ethnicity, and latitude. Confounder adjustment in this study does not include important morbidities (e.g., obesity) that are known to strongly influence both vitamin D and COVID-19 risk. The confounders considered, such as ethnicity and latitude are crudely estimated and are prone to misclassification. Although this study provides 
insight from a large cohort, there is high risk of bias from residual confounding.

Larger, more fully characterised, cohorts have enabled more thorough consideration of confounding variables. Three studies have used data from the UK Biobank to consider the association baseline (2006-2010) measurements of serum 25(OH)D and incident COVID-19. In analysis of the first release of UK Biobank data ( $n=265$ cases), Hastie et al. [33] demonstrated negative univariable associations between 25(OH)D and COVID-19 status. However, consistent with the considerations described above, the association was attenuated to the null in multivariable models considering important confounders, including ethnicity, sex, month of 25(OH)D measurement, deprivation, body mass index (BMI), smoking, self-reported health measures, and comorbidities such as hypertension and diabetes. These findings were confirmed by Raisi-Estabragh et al. [34] in a larger data set of the UK Biobank cohort ( $n=1326$ cases), demonstrating that amongst participants tested for COVID-19, there was no statistically significant association between seasonadjusted 25(OH)D levels and COVID-19 status in base models or with adjustment for age, sex, and ethnicity. Ma et al. [35] took a more extensive approach using the same data set, investigating the association of baseline serum $25(\mathrm{OH}) \mathrm{D}$, genetically predicted vitamin $\mathrm{D}$, and self-reported habitual vitamin D supplementation with COVID-19 test result in 8297 UK Biobank participants tested for COVID-19. Similar to Hastie et al. [33] and Raisi-Estabragh et al. [34] they found no association between serum 25(OH)D and COVID19 status, nor with genetically predicted vitamin D levels. They reported a protective association with habitual vitamin D supplementation (OR 0.66; 95\% CI 0.45-0.97). This is an interesting observation and the lack of consistency with serum and genetically determined vitamin D suggests that the observed protective effect from supplementation may be explained by confounding lifestyle and sociodemographic factors rather than vitamin D itself. However, a caveat with all three UK Biobank studies is that while they are prospective, which means that the COVID-19 cannot be responsible for the $25(\mathrm{OH}) \mathrm{D}$ measurement, the baseline $25(\mathrm{OH}) \mathrm{D}$ level was obtained approximately 10-14 years prior to COVID19 testing. There is some evidence of tracking of $25(\mathrm{OH})$ D across life [29], but there is of course the potential for $25(\mathrm{OH}) \mathrm{D}$ status to change over that time interval, as can supplementation habits.

A further cohort study in Israel, including 7,807 children and adults with at least one prior blood test for 25(OH)D and subsequent COVID-19 testing ( $n=782$ positive) [36], documented possible associations between 25(OH)D levels and COVID-19 disease. In multivariable logistic regression models categorising $25(\mathrm{OH}) \mathrm{D}$ status into vitamin $\mathrm{D}$ levels below or above $30 \mathrm{ng} / \mathrm{ml}, 25(\mathrm{OH}) \mathrm{D}$ in the lower category was associated with significantly greater odds of COVID-19 positivity, and in a separate model with greater odds of hospitalisation. However, in this analysis, the lack of knowledge about the time interval between 25(OH)D measurement and COVID-19 testing as well as the observational nature of the study limits the inferences that can be drawn from the findings.

Several hospital-based studies provide further results. Meltzer et al. [37] conducted a review of hospital-based cases, identifying all patients tested for COVID-19 with a record of serum $25(\mathrm{OH}) \mathrm{D}$ measurement in the preceding 12 months. They categorised patients into vitamin D deficient, sufficient, and uncertain, based on 25(OH)D levels and subsequent prescription history. In fully adjusted models, they report higher odds of COVID-19 in the vitamin D deficient group, compared to the vitamin D sufficient cohort. Given the observational study design, the same caveats of potential residual or unmeasured confounding described previously, together with issues due to selection bias from the sampling based on routine $25(\mathrm{OH}) \mathrm{D}$ measurement, are relevant. Maghbooli et al. [38] studied the association of serum 25(OH)D with COVID-19 severity in 235 COVID19 patients with $25(\mathrm{OH}) \mathrm{D}$ measured at the time of hospitalisation. They categorised vitamin D status into sufficient or deficient $(<30 \mathrm{ng} / \mathrm{ml})$. In a backward logistic regression model after adjusting for age, sex, BMI, smoking, and history of a chronic medical disorder, there were independent associations between vitamin D sufficiency and decreased disease severity. In a similar study, Mendes et al. [39] investigated 160 older adults admitted to a geriatric ward with COVID-19 and serum 25(OH)D measured during the acute illness. Outcomes were poor with $25 \%$ in-hospital morality in the whole sample, which disproportionately affected men (63\%). In sex-stratified multivariable Cox regression models they documented an independent association between lower vitamin D status and mortality in men, but not in women [40]. It is possible that the different relationship by sex reported by Mendes et al. reflects greater number of deaths in men than women, particularly as $25(\mathrm{OH}) \mathrm{D}$ levels were, in fact, higher in men. Luo et al. [41] present a single centre retrospective analysis from China, including 335 hospitalised COVID-19 patients and 560 age- and sex-matched controls. Serum 25(OH)D was measured at admission for cases and taken from within a defined 12 month period for the controls. Severity of COVID-19 was based on the level of respiratory involvement. In a general linear model adjusting for age, sex, BMI, and comorbidities, serum 25(OH)D was significantly lower among COVID-19 patient than in controls. In a multivariable logistic regression model, within the COVID-19 patients, vitamin D deficiency $(<30 \mathrm{nmol} / \mathrm{l})$ was associated with greater COVID-19 severity (OR: 2.72; $95 \%$ CI: $1.23,6.01, p<0.05)$. In a study of 222 hospitalised patients screened for SARS-CoV-2 infection, by Alguwaihes et al. [42], vitamin D status was not associated with 
infection risk. In multivariable logistic regression models, severe $25(\mathrm{OH}) \mathrm{D}$ deficiency $(<12.5 \mathrm{nmol} / \mathrm{l})$ was associated with increased mortality risk, although the association was not statistically significance [OR: 4.9 ; 95\% CI: 0.9, 25.8; $p=0.06]$. Importantly, in all of the aforementioned hospitalbased studies, $25(\mathrm{OH}) \mathrm{D}$ was measured at time of COVID19 hospitalisation, as vitamin D is an inverse acute phase reactant, with lower levels at times of physiological stress, these studies are at high risk of possible reverse causation. That is, that associations in these studies may be explained by greater levels of inflammation during acute illness reflecting more severe infection and causing a reduction in $25(\mathrm{OH})$ $\mathrm{D}$, rather than the other way around. Such effects have been demonstrated in humans for surgical procedures and trauma [16], and in animal models for infection [17], but a small recent study documented no apparent effect in the setting of human malaria infection [43, 44]. Of course, the fact that acute inflammation may drive a reduction in $25(\mathrm{OH}) \mathrm{D}$ levels does not preclude the lower concentrations also having a biological effect, but at present the physiological significance of this inverse acute phase response remains to be elucidated.

Confounding and reverse causation, therefore, may be important drivers of relationships reported in observational studies. In an attempt to mitigate these sources of spurious association, Butler-Laporte et al. [45] used a two sample Mendelian Randomisation study design to investigate evidence of a causal link between genetically determined serum 25(OH)D and COVID-19 susceptibility. Genetic instruments for $25(\mathrm{OH}) \mathrm{D}$ levels were identified from meta-analysis of two genome-wide association studies comprising a total of 443,734 participants of European Ancestry. Genetic variants linked to COVID-19 susceptibility were obtained from six genome-wide association studies from four countries. The authors report no clear effect of genetically determined 25(OH)D on COVID-19 susceptibility. Importantly, however, the Mendelian Randomisation design tests the association between outcome and a lifelong genetic component of the exposure, with the genetic instrument explaining a very small proportion of the exposure variance, so necessitating very large cohorts to achieve adequate statistical power. Where the exposure is likely to be thresholded, such as vitamin D status, the actual level of the exposure in the population is likely critical $[1,4]$ and such studies should not be viewed as substitutes for properly conducted RCTs.

\section{Interventional studies}

At the time of writing, there are very limited data from interventional studies. Annweiler et al. [46] report a quasiexperimental study of 77 elderly patients consecutively hospitalised for COVID-19. The patients were categorised into three groups including individuals who had received regular bolus vitamin $\mathrm{D}$ supplementation in the preceding year as ascertained from primary care records $(n=29)$, those who received a single oral dose of 80,000 IU vitamin D3 within hours of COVID-19 diagnosis $(n=16)$, and those who received no vitamin D supplementation $(n=32)$. They considered associations with 14-day mortality and a COVID-19 disease severity score, adjusting for a wide range of confounders. The authors document less severe COVID-19 and lower mortality in individuals taking vitamin D supplementation in the preceding year, but not in those supplemented after COVID-19 diagnosis. Assessment of survival benefit at 14 days is likely too early for observation of an effect from increment of vitamin D levels as a result of supplementation at time of COVID-19 diagnosis. Furthermore, the authors do not measure serum vitamin $\mathrm{D}$; therefore, associations described relate to recorded supplement use rather than directly measured vitamin D levels. In a similar study of 66 nursing home residents with COVID-19, Annweiller et al. [47] consider the relationship between vitamin D supplementation $(80,000 \mathrm{IU}$ vitamin D3 in the month preceding or the week following diagnosis of COVID-19) with COVID19 severity score or mortality over mean follow-up time of $36 \pm 17$ days. The authors document association of bolus vitamin D3 supplementation during or just before COVID19 with less severe COVID-19 and better survival rate. There was notable imbalance in sample size of the intervention $(n=57)$ and comparator groups $(n=9)$. Furthermore, the authors state that regular bolus supplementation (every 2-3 months) without measurement of serum vitamin D is recommended practice for nursing homes residents in the local setting (France). Given the absence of measured serum vitamin $\mathrm{D}$, the high likelihood that all study participants were receiving regular supplementation (even if this was not administered within the selected window of this study), and the markedly imbalanced samples in the intervention and comparator groups, it is possible that the observed associations may be subject to statistical artefact.

Tan et al. [48] reported a quasi-experimental study exploiting a change in treatment protocols for COVID-19 patients in their hospital (Singapore General Hospital) from no supplementation to routine administration of a vitamin $\mathrm{D}$, magnesium, and vitamin B12 combination supplement. Consecutive patients admitted after the change in policy were taken as the intervention group $(n=17)$, and those prior as comparators $(n=26)$. They included patients aged over 50 years hospitalised with COVID-19 and not requiring oxygen therapy or intensive care. They report significantly higher proportion of patients with clinical deterioration (oxygen therapy or intensive care) in the control $(n=16)$ than in the interventional group $(n=3)$. These results suggest a possible protective effect from supplementation. However, as a combined supplementation regimen was used, it is not possible to attribute any associations to a single agent. In addition, the interventional group comprised a cohort treated 
later on in the pandemic, it is likely that other aspects of their care may have improved, compared to the earlier patients used as controls, with increased experience of treating physicians with COVID-19. The small number of participants and events limits confounder adjustment in this analysis. On balance, there are multiple factors that preclude definitive attribution of the observed differences in this study to vitamin D supplementation.

Rastogi et al. [49] undertook a small randomised trial of the effect of high-dose short-term vitamin D supplementation on COVID-19 outcomes. They randomised 40 patients with polymerase chain reaction (PCR) confirmed SARS-CoV-2 to receive cholecalciferol 60,000 IU daily $(n=16)$ until achieving serum 25(OH)D $>50 \mathrm{ng} / \mathrm{l}$ (tested at day 7 and day 14), vs placebo $(n=24)$. PCR tests for SARS-CoV-2 were performed at 7-day intervals (7, 14, and 21 days). The authors report that, during the study period, 10 out of $16(62.5 \%)$ participants in the intervention group achieved SARS-CoV-2 negativity compared to 5 out of 24 $(20.8 \%)$ participants $(p=0.018)$ in the control arm. The mean duration to SARS-CoV-2 negativity was $17.6 \pm 6.1$ and 17.6 \pm 6.4 days $(p=0.28)$ in the intervention and control arm, respectively. The randomisation method is not documented, and no reason is given for the unequal allocation to active and placebo groups. The intervention was not blinded, and analytical approach ("modified intention to treat") is obscure. While the authors imply that a lower duration to SARS-CoV-2 negativity indicates more rapid viral clearance, data on severity of illness or time to discharge are not reported. All of these considerations, together with the very small size of this trial, substantially reduce any confidence in these findings.

Entrenas Castillo et al. [50] present pilot results from a RCT of the effect of calcifediol (25-hydroxy-vitamin D) supplementation on intensive care admission and death in a hospitalised cohort of 76 adults with PCR confirmed SARS-CoV-2 infection and radiographic evidence of viral pneumonia. There was "electronic" randomisation of consecutive eligible participants on admission at a ratio of 2:1 to intervention. Supplementation with calcifediol was undertaken on admission, day 3 , day 7 , and weekly thereafter at an initial dose of $0.532 \mathrm{mg}$ and $0.266 \mathrm{mg}$ for subsequent doses. The control group received standard medical care. There was no placebo pill for the controls. Calcifediol supplementation was stopped if a patient was admitted to intensive care. Of the 50 patients receiving calciferol, one $(2 \%)$ required intensive care, compared to $50 \%(n=13)$ in the control cohort $(p<0.001)$. There were no deaths in the intervention arm, compared with two deaths in the control group. These results suggest beneficial effects of calciferol supplementation in reducing severity of COVID-19 course. However, the results are much more dramatic than is biologically plausible, which raises the possibility that the observed effects are significantly inflated by systematic bias. Indeed, due to the small sample, despite randomisation, there is heterogeneous distribution of important morbidities such as hypertension and diabetes with greater rates of disease in the control group.

Murai et al. [51] undertook a more robustly conducted RCT from Brazil. They tested the effect of a single high dose of oral vitamin D (200,000 IU) on clinical outcomes of patients hospitalised with moderate-severe COVID-19 (PCR confirmed). Patients were randomly assigned to intervention $(n=120)$ or placebo $(n=120)$. Randomisation was allocated using a computer-generated code. Outcomes were defined a priori. The primary outcome was length of stay, a set of secondary in-hospital outcomes were also pre-specified. The authors reported no differences in length of stay, in-hospital mortality, admission to intensive care, or requirement for mechanical ventilation between the intervention and control groups. Contrary to the findings of Entrenas Castillo et al. [50] and Rastogi et al. [49], the findings from this larger and better conducted trial [51] do not support a role for vitamin D supplementation for treatment of moderate-severe COVID-19. However, given the still modest sample sizes, an effect is not excluded by these findings and further studies in larger populations with appropriately matched controls are needed, with randomised, blinded intervention designs as the optimal approach, albeit one which may be difficult to achieve given the practical considerations.

\section{Current guidance for vitamin $D$ therapy in the context of COVID-19}

In response to the discussion around the potential preventative and therapeutic role of vitamin D in COVID-19, public health agencies in the UK published three rapid evidence reviews in June 2020. A report conducted by the National Institute for Health and Care Excellence (NICE) and another by the Royal Society were specifically concerned with the role of vitamin D in the context of COVID-19 [52, 53]. A third report from the Scientific Advisory Committee on Nutrition (SACN) covered respiratory infections other than COVID-19 [54]. Furthermore, joint international guidance on vitamin D supplementation in the context of COVID19 has also been published from the American Society for Bone and Mineral Research (ASBMR), Endocrine Society, American Association of Clinical Endocrinologists (AACE), European Calcified Tissue Society (ECTS), the National Osteoporosis Foundation (NOF), and the International Osteoporosis Foundation (IOF) [55]. These reports predominantly conclude that there is insufficient evidence to recommend use of vitamin $\mathrm{D}$ for treatment or prevention of respiratory tract infections or COVID-19 but endorse supplementation for maintenance of musculoskeletal health. 
There is considerable variation between existing national guidelines, both in relation to recommended dietary intakes and minimum serum levels of vitamin D. The UK SACN report in 2016 used $25 \mathrm{nmol} / 125(\mathrm{OH}) \mathrm{D}$ as the threshold for defining the recommended nutrient intake (RNI) of $400 \mathrm{IU}$ per day [56]. Groups at high risk of vitamin D insufficiency, and many individuals in the winter months, may require supplementation to achieve sufficient levels. At the time of writing, the UK government has issued free vitamin D supplements at $400 \mathrm{IU}$ daily for care home residents and other vulnerable groups; while this dose is usually adequate to prevent severe deficiency in the context of population health, it may not provide reliable repletion in those at high risk of deficiency. However, it is important to note that SACN's remit is risk assessment for deficiency of a nutrient at the population level, rather than optimum levels in individuals or recommendations for clinical treatment. Other guidelines suggest that higher doses, e.g., 800-3200 IU per day, may be required in this context to achieve repletion before settling on an appropriate lower maintenance dose $[53,54,57$, 58]. Indeed there have been recent calls for the UK RNI to be raised to 800 IU daily given the potential signals for benefit and absence of any safety concerns at this intake [59]. EU guidance suggests an intake of 600 IU daily and that from the US suggests $600 \mathrm{IU}$, or in those aged over 70 years, $800 \mathrm{IU}$ daily [60-62]. Importantly, there is no evidence for additional benefit of pharmacological "mega-doses"; indeed such approaches have been associated with increased risk of falls and fractures [63], and of overt vitamin D toxicity [64].

\section{Ongoing and future work}

The prevailing evidence is such that a benefit of vitamin $\mathrm{D}$ supplementation in the prevention or treatment of COVID19 disease cannot be proved or refuted. The underlying biology suggests a role for vitamin $\mathrm{D}$ in modulating host responses to several respiratory pathogens $[1,65,66]$, but whether these local cellular effects of $1,25(\mathrm{OH})_{2}$-vitamin D may be usefully influenced by vitamin D supplementation is uncertain. Thus, further work, including robustly designed and conducted RCTs, are needed. Queen Mary University of London has initiated a prospective national study, COVIDENCE UK, recruiting more than 18,000 participants [67]. In an initial online questionnaire information is being collected on determinants of vitamin D status and other important risk factors. These data are linked to notifications of incident COVID-19 identified from monthly online follow-up, cross-checked against routinely collected health outcome data held by NHS Digital. The "CORONAVIT" RCT, recruiting 6200 individuals, randomised in an open label design to either the UK recommendation of $400 \mathrm{IU} /$ day (control), or $25(\mathrm{OH}) \mathrm{D}$ testing with replacement at a dose of $800 \mathrm{IU}$ or $3200 \mathrm{IU} /$ day is underway to investigate the potential for different vitamin D supplementation strategies to reduce the risk or severity of COVID-19 [68]. Several other trials are also underway or planned to investigate the preventative or therapeutic potential of vitamin D supplementation in the context of COVID-19 (Table 1).

\section{Conclusion}

Although there are a growing number of studies into the links between vitamin D and COVID-19, they are mostly small observational studies with high risk of bias, residual confounding and reverse causality. There is need for adequately powered clinical trial data to more definitively investigate the role of vitamin D in COVID-19. At present, evidence to support vitamin D supplementation for the prevention or treatment of this infection is inconclusive. It seems likely, given shielding advice to individuals clinically vulnerable to severe COVID-19, that many frail elderly individuals will have experienced musculoskeletal deconditioning and reduced $25(\mathrm{OH}) \mathrm{D}$ status as a result of remaining indoors throughout the summer months (personal communication, UK Royal Osteoporosis Society). While the current evidence base does not support supplementation with vitamin $\mathrm{D}$ alone as a population health measure to prevent fracture [1,69], it is clear that supplementation will reduce the risk of overt manifestations of vitamin D deficiency such as osteomalacia [1]. In this context, the supplemental strategy at the level of the population differs to that targeted to those overtly vitamin D deficient, but the evidence base clearly demonstrates that use of pharmacological mega-dose vitamin D is associated with no additional benefit but has potential for harm. Thus, in the absence of any further compelling data, adherence to current guidance on vitamin D supplementation (varying from $400 \mathrm{IU}$ daily as the UK RNI up to $800 \mathrm{IU}$ per day in the US population), usually predicated principally on maintaining musculoskeletal health, appears appropriate. 


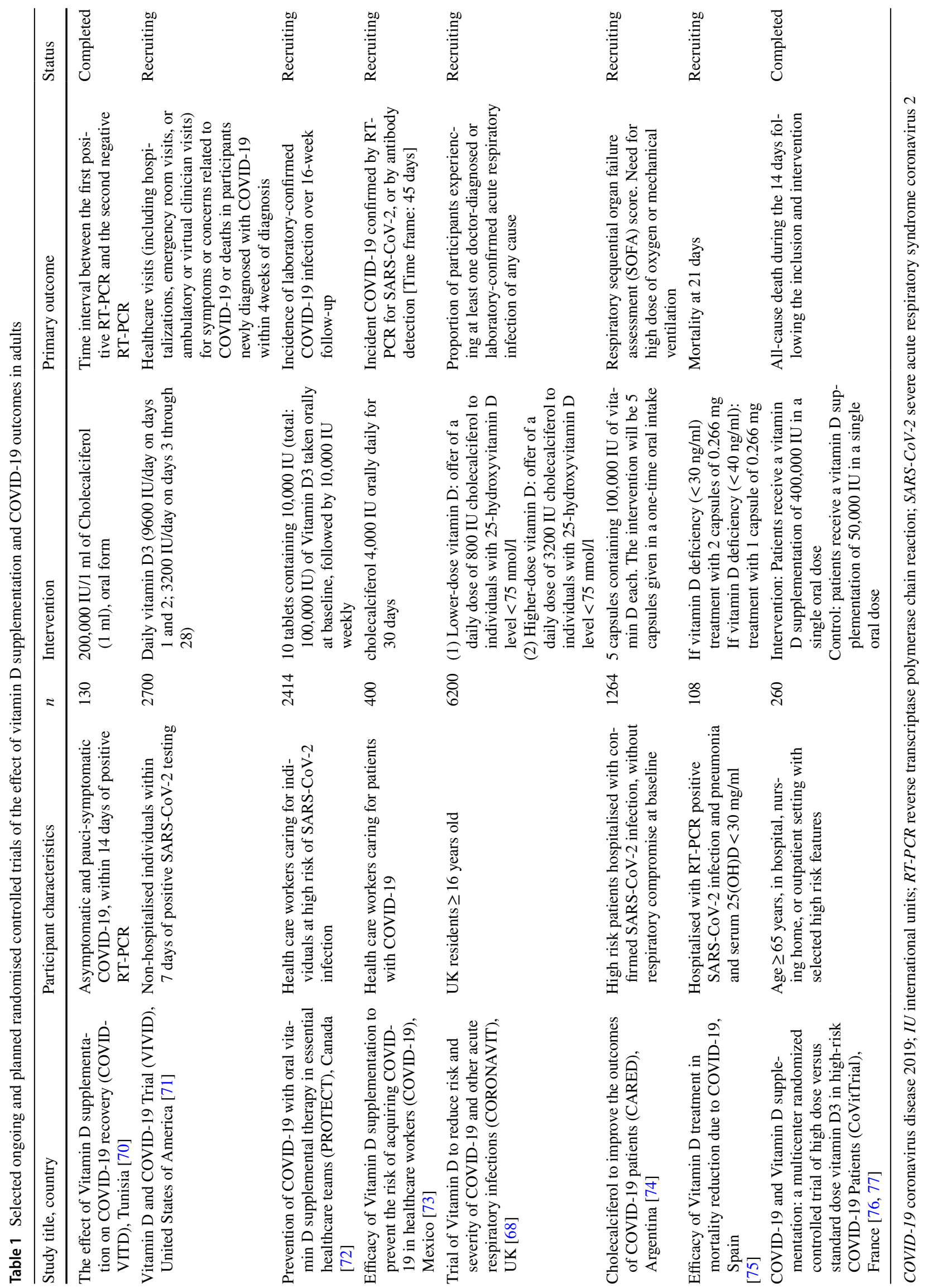


Acknowledgements We would like to thank the Medical Research Council (UK), National Institute for Health Research (NIHR), Wellcome Trust, Versus Arthritis, Royal Osteoporosis Society Osteoporosis and Bone Research Academy and International Osteoporosis Foundation for supporting this work. SEP and PBM acknowledges support from the NIHR Barts Biomedical Research Centre. ZRE was supported by British Heart Foundation Clinical Research Training Fellowship No. FS/17/81/33318.

Funding There was no direct funding for this review.

\section{Declarations}

Conflict of interest $\mathrm{NCH}$ reports personal fees, consultancy, lecture fees and honoraria from Alliance for Better Bone Health, AMGEN, MSD, Eli Lilly, Servier, Shire, UCB, Consilient Healthcare, Kyowa Kirin and Internis Pharma, outside the submitted work. ZRE reports no conflicts of interest. ARM reports grant support for the CORONAVIT trial from Pharma Nord Ltd, Synergy Biologics Ltd, Cytoplan Ltd, and DSM Ltd, outside the submitted work. RJM has no disclosures. EMC reports conference support and lecture fees from Eli Lilly, UCB and Amgen, outside the submitted work. KAW reports lecture fees from Abbott Nutrition and Pfizer Healthcare, outside the submitted work. ALD was an expert advisor on the NICE Vitamin D and COVID-19 evidence review (2020). SLN is a member of SACN Main Committee and was on the SACN Vitamin D working group responsible for setting the new vitamin D recommendations for the UK (2010-2016); contributing to the SACN ARTI Report and the NICE Vitamin D and COVID-19 Report. SLN also declares reports honorariums received from Thornton Ross and the US Council for Responsible Nutrition on vitamin D, both of which have been donated to charities. CC, PBM, and SEP declare that they have no conflict of interest.

Ethical approval/informed consent Ethics review and informed consent are not applicable.

Statement of human and animal rights There was no direct involvement of patients or participants in this literature review.

Open Access This article is licensed under a Creative Commons Attribution 4.0 International License, which permits use, sharing, adaptation, distribution and reproduction in any medium or format, as long as you give appropriate credit to the original author(s) and the source, provide a link to the Creative Commons licence, and indicate if changes were made. The images or other third party material in this article are included in the article's Creative Commons licence, unless indicated otherwise in a credit line to the material. If material is not included in the article's Creative Commons licence and your intended use is not permitted by statutory regulation or exceeds the permitted use, you will need to obtain permission directly from the copyright holder. To view a copy of this licence, visit http://creativecommons.org/licenses/by/4.0/.

\section{References}

1. Harvey NC, Biver E, Kaufman JM et al (2017) The role of calcium supplementation in healthy musculoskeletal ageing: an expert consensus meeting of the European Society for Clinical and Economic Aspects of Osteoporosis, Osteoarthritis and Musculoskeletal Diseases (ESCEO) and the International Foundation for Osteoporosis (IOF). Osteoporos Int 28:447-462

2. Bikle DD (2009) Vitamin D and immune function: understanding common pathways. Curr Osteoporos Rep 7:58-63
3. Prietl B, Treiber G, Pieber TR, Amrein K (2013) Vitamin D and immune function. Nutrients 5:2502-2521

4. Bikle DD (2014) Vitamin D metabolism, mechanism of action, and clinical applications. Chem Biol 21:319-329

5. Fiorino S, Gallo C, Zippi M et al (2020) Cytokine storm in aged people with CoV-2: possible role of vitamins as therapy or preventive strategy. Aging Clin Exp Res 32:2115-2131

6. Daneshkhah A, Agrawal V, Eshein A et al (2020) Evidence for possible association of vitamin D status with cytokine storm and unregulated inflammation in COVID-19 patients. Aging Clin Exp Res 32:2141-2158

7. Mandal AKJ, Baktash V, Hosack T, Missouris CG (2020) Vitamin D status and COVID-19 in older adults. Aging Clin Exp Res $32: 2425-2426$

8. Skrobot A, Demkow U, Wachowska M (2018) Immunomodulatory role of vitamin D: a review. Adv Exp Med Biol 1108:13-23

9. Maretzke F, Bechthold A, Egert S et al (2020) Role of vitamin $\mathrm{D}$ in preventing and treating selected extraskeletal diseases-an umbrella review. Nutrients 12:969

10. Bischoff-Ferrari HA, Vellas B, Rizzoli R et al (2020) Effect of vitamin D supplementation, omega-3 fatty acid supplementation, or a strength-training exercise program on clinical outcomes in older adults: the DO-HEALTH Randomized Clinical Trial. JAMA 324:1855-1868

11. Cobbold P (2020) Rapid response BMJ—we need to understand the cause to tackle the risks. https://www.bmj.com/content/371/ bmj.m3790/rr. Accessed 28 Nov 2020

12. Aranow C (2011) Vitamin D and the immune system. J Investig Med 59:881-886

13. Lanham-New SA, Webb AR, Cashman KD et al (2020) Vitamin D and SARS-CoV-2 virus/COVID-19 disease. BMJ Nutr Prev Health 13:106-110

14. Harvey NC, Cooper C (2012) Vitamin D: Some perspective please. BMJ 345:e4695

15. Vimaleswaran KS, Berry DJ, Lu C et al (2013) Causal relationship between obesity and vitamin $\mathrm{D}$ status: bi-directional Mendelian randomization analysis of multiple cohorts. PLoS Med 10:e1001383

16. Waldron JL, Ashby HL, Cornes MP et al (2013) Vitamin D: a negative acute phase reactant. J Clin Pathol 66:620-622

17. Nonnecke BJ, McGill JL, Ridpath JF et al (2014) Acute phase response elicited by experimental bovine diarrhea virus (BVDV) infection is associated with decreased vitamin D and E status of vitamin-replete preruminant calves. J Dairy Sci 97:5566-5579

18. de Souza WN, Norde MM, Oki É et al (2016) Association between 25 -hydroxyvitamin $\mathrm{D}$ and inflammatory biomarker levels in a cross-sectional population-based study, São Paulo, Brazil. Nutr Res 36:1-8

19. Czarnik T, Czarnik A, Gawda R et al (2018) Vitamin D kinetics in the acute phase of critical illness: a prospective observational study. J Crit Care 43:294-299

20. Rizzoli R (2021) Vitamin D supplementation: upper limit for safety revisited? Aging Clin Exp Res 33:19-24

21. Jolliffe DA, Camargo CA, Sluyter JD et al (2021) Vitamin D supplementation to prevent acute respiratory infections: a systematic review and meta-analysis of aggregate data from randomised controlled trials. Lancet Diabetes Endocrinol 9:276-292

22. Vuichard Gysin D, Dao D, Gysin CM et al (2016) Effect of vitamin D3 supplementation on respiratory tract infections in healthy individuals: a systematic review and meta-analysis of randomized controlled trials. PLoS ONE 11:e0162996

23. Mok CK, Ng YL, Ahidjo BA et al (2020) Calcitriol, the active form of vitamin $\mathrm{D}$, is a promising candidate for COVID-19 prophylaxis. bioRxiv 2020.06.21.162396. https://doi.org/10. $1101 / 2020.06 .21 .162396$ 
24. Ilie PC, Stefanescu S, Smith L (2020) The role of vitamin D in the prevention of coronavirus disease 2019 infection and mortality. Aging Clin Exp Res 32:1195-1198

25. Laird E, Rhodes J, Kenny RA (2020) Vitamin D and inflammation: potential implications for severity of Covid-19. Ir Med J 113:81-88

26. Walrand S (2021) Autumn COVID-19 surge dates in Europe correlated to latitudes, not to temperature-humidity, pointing to vitamin D as contributing factor. Sci Rep 11:1981

27. Singh S, Kaur R, Singh RK (2020) Revisiting the role of vitamin $\mathrm{D}$ levels in the prevention of COVID-19 infection and mortality in European countries post infections peak. Aging Clin Exp Res 32:1609-1612

28. Greenland S, Robins J (1994) Invited commentary: ecologic studies-biases, misconceptions, and counterexamples. Am J Epidemiol 139:747-760

29. Wise SA, Phinney KW, Tai SSC et al (2017) Baseline assessment of 25-hydroxy vitamin D assay performance: a vitamin D standardization program (VDSP) interlaboratory comparison study. J AOAC Int 100:1244-1252

30. D'Avolio A, Avataneo V, Manca A et al (2020) 25-hydroxyvitamin D concentrations are lower in patients with positive PCR for SARS-CoV-2. Nutrients 12:1359

31. Fasano A, Cereda E, Barichella M et al (2020) COVID -19 in Parkinson's disease patients living in Lombardy, Italy. Mov Disord 35:1089-1093

32. Kaufman HW, Niles JK, Kroll MH et al (2020) SARS-CoV-2 positivity rates associated with circulating 25 -hydroxyvitamin D levels. PLoS ONE 15:e0239252

33. Hastie CE, Mackay DF, Ho F et al (2020) Vitamin D concentrations and COVID-19 infection in UK Biobank. Diabetes Metab Syndr Clin Res Rev 14:561-565

34. Raisi-Estabragh Z, Mccracken C, Bethell MS et al (2020) Greater risk of severe COVID-19 in Black, Asian and Minority ethnic populations is not explained by cardiometabolic, socioeconomic or behavioural factors, or by $25(\mathrm{OH})$-vitamin D status: study of 1326 cases from the UK Biobank. J Public Health 25:1-10

35. Ma H, Zhou T, Heianza Y, Qi L (2021) Habitual use of vitamin D supplements and risk of coronavirus disease 2019 (COVID-19) infection: a prospective study in UK Biobank. Am J Clin Nutr 113:1275-1281

36. Merzon E, Tworowski D, Gorohovski A et al (2020) Low plasma $25(\mathrm{OH})$ vitamin $\mathrm{D}$ level is associated with increased risk of COVID-19 infection: an Israeli population-based study. FEBS J 287:3693-3702

37. Meltzer DO, Best TJ, Zhang $\mathrm{H}$ et al (2020) Association of vitamin D status and other clinical characteristics with COVID-19 test results. JAMA Netw Open 3:e2019722

38. Maghbooli Z, Sahraian MA, Ebrahimi M et al (2020) Vitamin $D$ sufficiency, a serum 25-hydroxyvitamin D at least $30 \mathrm{ng} /$ $\mathrm{mL}$ reduced risk for adverse clinical outcomes in patients with COVID-19 infection. PLoS ONE 15:1-13

39. Mendes A, Serratrice C, Herrmann FR et al (2020) Predictors of In-hospital mortality in older patients with COVID-19: the COVID age study. J Am Med Dir Assoc 21:1546-1554.e3

40. Hars M, Mendes A, Serratrice C et al (2020) Sex-specific association between vitamin D deficiency and COVID-19 mortality in older patients. Osteoporos Int 31:2495-2496

41. Luo X, Liao Q, Shen Y et al (2021) Vitamin D deficiency is inversely associated with COVID-19 incidence and disease severity in Chinese people. J Nutr 151:98-103

42. Alguwaihes AM, Sabico S, Hasanato R et al (2021) Severe vitamin D deficiency is not related to SARS-CoV-2 infection but may increase mortality risk in hospitalized adults: a retrospective case-control study in an Arab Gulf country. Aging Clin Exp Res $33: 1415-1422$

43. Jolliffe DA, Stefanidis C, Wang Z et al (2020) Vitamin D metabolism is dysregulated in asthma and chronic obstructive pulmonary disease. Am J Respir Crit Care Med 202:371-382

44. Newens K, Filteau S, Tomkins A (2006) Plasma 25-hydroxyvitamin D does not vary over the course of a malarial infection. Trans R Soc Trop Med Hyg 100:41-44

45. Butler-Laporte G, Nakanishi T, Mooser V et al (2020) Vitamin D and Covid-19 susceptibility and severity: a Mendelian randomization study. medRxiv https://doi.org/10.1101/2020.09.08.20190975

46. Annweiler G, Corvaisier M, Gautier J et al (2020) Vitamin D supplementation associated to better survival in hospitalized frail elderly covid-19 patients: the geria-covid quasi-experimental study. Nutrients 12:1-12

47. Annweiler C, Hanotte B, Grandin de l'Eprevier C et al (2020) Vitamin D and survival in COVID-19 patients: a quasi-experimental study. J Steroid Biochem Mol Biol 204:1-6

48. Tan CW, Ho LP, Kalimuddin S et al (2020) Cohort study to evaluate effect of vitamin D, magnesium, and vitamin B12 in combination on severe outcome progression in older patients with coronavirus (COVID-19). Nutrition 79-80:111017

49. Rastogi A, Bhansali A, Khare N et al (2020) Short term, high-dose vitamin D supplementation for COVID-19 disease: a randomised, placebo-controlled, study (SHADE study). Postgrad Med J. https://doi.org/10.1136/postgradmedj-2020-139065. Online ahead of print.

50. Entrenas Castillo M, Entrenas Costa LM, Vaquero Barrios JM et al (2020) Effect of calcifediol treatment and best available therapy versus best available therapy on intensive care unit admission and mortality among patients hospitalized for COVID-19: a pilot randomized clinical study. J Steroid Biochem Mol Biol 203:105751

51. Murai IH, Fernandes AL, Sales LP et al (2021) Effect of a single high dose of vitamin D3 on hospital length of stay in patients with moderate to severe COVID-19: a randomized clinical trial. JAMA 325:1053-1060

52. National Institute for Health and Care Excellence (2020) Evidence review vitamin D for COVID-19. https://www.nice.org.uk/guida nce/ng187. Accessed 18 May 2021

53. The Royal Society (2020) Vitamin D deficiency and COVID-19

54. Scientific Advisory Committee on Nutrition (SACN) (2020) Update of rapid review: vitamin D and acute respiratory tract infections. December 2020. https://assets.publishing.service. gov.uk/government/uploads/system/uploads/attachment_data/ file/945179/SACN_December2020_VitaminD_AcuteRespirator yTractInfections.pdf. Accessed 18 May 2021

55. Joint Guidance on Vitamin D in the Era of COVID-19 from the ASBMR, AACE, Endocrine Society, ECTS, NOF, and IOF American Society for Bone and Mineral Research (2020) Am. Soc. Bone Miner. Res. https://www.asbmr.org/about/statementdetail/joint-guidance-on-vitamin-d-in-the-era-of-covid-19. Accessed 8 Jan 2021

56. Public Health England (2016) Vitamin D and health 2016. Scientific Advisory Committee on Nutrition (SACN). https://www. gov.uk/government/groups/scientific-advisory-committee-on-nutri tion. Accessed 18 May 2021

57. National Institute for Health and Care Excellence (2020) COVID19 rapid guideline: vitamin D. https://www.nice.org.uk/guidance/ ng187. Accessed 18 May 2021

58. Statement from PHE and NICE on vitamin D supplementation during winter-GOV.UK. https://www.gov.uk/government/publi cations/vitamin-d-supplementation-during-winter-phe-and-nicestatement/statement-from-phe-and-nice-on-vitamin-d-supplement ation-during-winter. Accessed 14 Dec 2020 
59. Griffin G, Hewison M, Hopkin J et al (2020) Preventing vitamin D deficiency during the COVID-19 pandemic: UK definitions of vitamin D sufficiency and recommended supplement dose are set too low. Clin Med 21:e48-e51

60. Bouillon R (2017) Comparative analysis of nutritional guidelines for vitamin D. Nat Rev Endocrinol 13:466-479

61. Ross AC, Taylor CL, Yaktine AL, Del Valle HB (eds) (2011) Institute of Medicine (US) Committee to review dietary reference intakes for vitamin D and calcium. Dietary reference intakes for calcium and vitamin D. National Academies Press (US), Washington (DC). PMID: 21796828

62. EFSA Panel on Dietetic Products, Nutrition and Allergies (NDA) (2016) Dietary reference values for vitamin D. https://doi.org/10. 2903/j.efsa.2016.4547. Accessed 18 May 2021

63. Sanders KM, Stuart AL, Williamson EJ et al (2010) Annual highdose oral vitamin D and falls and fractures in older women: a randomized controlled trial. JAMA 303:1815-1822

64. Galior K, Grebe S, Singh R (2018) Development of vitamin D toxicity from overcorrection of vitamin $\mathrm{D}$ deficiency: a review of case reports. Nutrients 10:953

65. Greiller CL, Martineau AR (2015) Modulation of the immune response to respiratory viruses by vitamin D. Nutrients $7: 4240-4270$

66. Zdrenghea MT, Makrinioti H, Bagacean C et al (2017) Vitamin $\mathrm{D}$ modulation of innate immune responses to respiratory viral infections. Rev Med Virol 27:1-12

67. Queen Mary University of London. Covidence UK. https://www. qmul.ac.uk/covidence/. Accessed 26 Nov 2020

68. Trial of vitamin D to reduce risk and severity of COVID-19 and other acute respiratory infections-full text view-ClinicalTrials.gov. https://clinicaltrials.gov/ct2/show/NCT04579640?term $=$ vitamin + D\&cond $=$ Covid $19 \&$ draw $=3$. Accessed 18 May 2021

69. Trajanoska K, Morris JA, Oei L et al (2018) Assessment of the genetic and clinical determinants of fracture risk: genome wide association and Mendelian randomisation study. BMJ 362:1-14

70. The effect of vitamin D supplementation on COVID-19 recovery-full text view-ClinicalTrials.gov. https://clinicaltrials.gov/ ct2/show/NCT04883203?term=vitamin+D\&cond=Covid19\& draw $=3 \&$ rank $=1$. Accessed 18 May 2021
71. Vitamin D and COVID-19 trial-full text view-ClinicalTrials. gov. https://clinicaltrials.gov/ct2/show/NCT04536298?term= vitamin + D\&cond $=$ Covid $19 \&$ draw $=3 \&$ rank $=4$. Accessed 18 May 2021

72. Prevention of COVID-19 with oral vitamin D supplemental therapy in essential healthcare teams-full text view-ClinicalTrials.gov. https://clinicaltrials.gov/ct2/show/NCT04483635?term= vitamin + D\&cond $=$ Covid $19 \&$ draw $=3 \&$ rank $=8$. Accessed 18 May 2021

73. Efficacy of vitamin D supplementation to prevent the risk of acquiring COVID-19 in healthcare workers-full text viewClinicalTrials.gov. https://clinicaltrials.gov/ct2/show/NCT04 535791 ?term $=$ vitamin + D\&cond $=$ Covid $19 \&$ draw $=3$. Accessed 18 May 2021

74. Cholecalciferol to improve the outcomes of COVID-19 patientsfull text view-ClinicalTrials.gov. https://clinicaltrials.gov/ct2/ show/NCT04411446?term $=$ vitamin + D\&cond $=$ Covid19\&draw $=$ $3 \&$ rank $=25$. Accessed 18 May 2021

75. Efficacy of vitamin D treatment in mortality reduction due to COVID-19-full text view-ClinicalTrials.gov. https://clinicaltr ials.gov/ct2/show/NCT04621058?term $=$ vitamin + D\& cond $=$ Covid19\&draw $=3 \&$ rank $=38$. Accessed 18 May 2021

76. Covid-19 and vitamin D supplementation: a multicenter randomized controlled trial of high dose versus standard dose vitamin D3 in high-risk COVID-19 patients (CoVitTrial)—full text view-ClinicalTrials.gov. https://clinicaltrials.gov/ct2/show/ NCT04344041 term $=$ vitamin + D\& cond $=$ Covid $19 \&$ draw $=3 \&$ rank=43. Accessed 18 May 2021

77. Annweiler C, Beaudenon M, Gautier J et al (2020) COvid-19 and high-dose VITamin D supplementation TRIAL in high-risk older patients (COVIT-TRIAL): study protocol for a randomized controlled trial. Trials 21:1031

Publisher's Note Springer Nature remains neutral with regard to jurisdictional claims in published maps and institutional affiliations. 